\title{
Multiple asymptomatic tricuspid papillary Fibroelastomas - Case Report
}

\author{
Miguel Tapia-Sansores ${ }^{1}$, Mara Escudero-Salamanca ${ }^{1}$, Ana-Valentina Gutierrez-Solana-Ossa ${ }^{1}$, Joaquin Berarducci ${ }^{1}$, Javier Ivan Armenta- \\ Moreno $^{1}$ and Nilda Espinola-Zavaleta ${ }^{1,2 *}$ \\ ${ }^{1}$ Department of Nuclear Cardiology, National Institute of Cardiology Ignacio Chavez, Mexico City, Mexico \\ ${ }^{2}$ Department of Echocardiography, ABC Medical Center, Mexico City, Mexico
}

\begin{abstract}
Papillary fibroelastomas (PFE) are the third most common primary cardiac tumor with an incidence of up to $0.33 \%$ in autopsy series. Most patients with PFE are clinically asymptomatic with isolated tumors; multiple PFE are rare. We present a case of a 70-year-old man with cardiovascular risk factors and ischemic heart disease, whom during echocardiographic assessment multiple pediculated, highly mobile and echo-dense masses in the tricuspid valve were incidentally identified. He was afebrile and had no clinical signs or biochemical evidence suggesting infective endocarditis. In the 7 year-follow-up, the patient remains asymptomatic without modifications of the diameter of the tricuspid PFE in the annual routine echocardiographic study.
\end{abstract}

\section{Introduction}

Primary heart tumors are extremely rare across all age groups with a reported prevalence of $0.001 \%$ to $0.03 \%$ in autopsy series [1]. Papillary fibroelastomas (PFE) are the third most common primary cardiac tumor with an incidence of up to $0.33 \%$ in autopsy series [2]. It has no spontaneous regression, and may have focal calcification with occasionally cystic degeneration [3]. Most patients with PFE are clinically asymptomatic, however symptomatic patients may debut with transient ischemic attacks, strokes, angina, myocardial infarctions, and dyspnea. More than half of the symptomatic patients have cerebral embolic symptoms [2].

Cardiac PFE are usually attached to valvular or mural endocardium [4]. Transesophageal echocardiography is the recommended imaging modality for diagnosis and characterization of PFE, with a sensitivity of $89 \%$ and specificity of $88 \%$ in lesions measuring $2 \mathrm{~cm}$ or more [5]. Cardiac Magnetic Resonance (CMR) can differentiate tumors and thrombi [3]. PFE can be found anywhere in the heart, but $80-90 \%$ are found in valvular endocardium [5]. The aortic valve is the most common site (37-45\%), and in a minor percentage the other valves but rarely in the left atrial or ventricular endocardium [2,5-7]. Single PFE are found in $91 \%$ and the rest are multiple from different endocardium sites [8,9].

Treatment of PFE is surgical resection of the tumor, shaving from the valvular leaflets with reconstruction or replacement of the valve $[3,5,10]$. Resection should be considered for patients with tumors larger than $1 \mathrm{~cm}$, or mobile-type PFE $[4,5,10]$. In asymptomatic patients, the surgical management requires a thorough analysis of the benefits and the possible consequences of a surgical intervention. The prognosis for patients with PFE treated surgically is excellent, with no reported cases of recurrence [5].

\section{Case Report}

70-year-old man with multiple PFE in the tricuspid valve, that were identified during pre-surgical evaluation for coronary artery bypass grafting. Medical history showed well controlled Type II diabetes, hypertension, benign prostatic hyperplasia and a first-degree atrioventricular block. The patient was diagnosed with asymptomatic coronary artery disease during a routine check-up evaluation, where an abnormal stress test was identified suggesting myocardial ischemia. The coronary angiography detected significant lesions in the left anterior descending, circumflex and right coronary arteries. Therefore, a coronary artery bypass graft was successfully performed without complications. He was afebrile and had no clinical signs or biochemical evidence suggesting infective endocarditis.

Pre-surgical echocardiographic evaluation showed a cardiac mass in the middle portion of the anterior tricuspid leaflet of $1.09 \mathrm{~cm} \mathrm{x} 0.98$ $\mathrm{cm}$ of diameter, pediculated, homogenous echo-dense, and highly mobile as well as two other smaller masses in the septal leaflet and tricuspid subvalvular apparatus, which contributed to a mild tricuspid regurgitation (Figure 1, video 1). A mild dilation of the right cavities was identified, and the right systolic ventricular function was depressed (TAPSE - $14 \mathrm{~mm}$ ). The pulmonary arterial systolic pressure was normal $(21 \mathrm{mmHg})$, also the left ventricular systolic function $(\mathrm{LVEF}=73 \%)$, and a mild mitral regurgitation was detected.

In the 7 year-follow-up, the patient remains asymptomatic with adequate control of his comorbidities, without modifications of the diameter of the tricuspid PFE in the annual routine echocardiographic study.

${ }^{*}$ Correspondence to: Nilda Espinola-Zavaleta, MD, PhD, Department of Nuclear Cardiology, National Institute of Cardiology Ignacio Chávez, Juan Badiano No 1, Colonia Sección XVI, P.C-14080, Tlalpan, Mexico City, Mexico, E-mail: niesza2001@hotmail.com

Key words: papillary fibroelastoma, echocardiography, tricuspid valve

Received: February 09, 2021; Accepted: February 16, 2021; Published: February 19,2021 

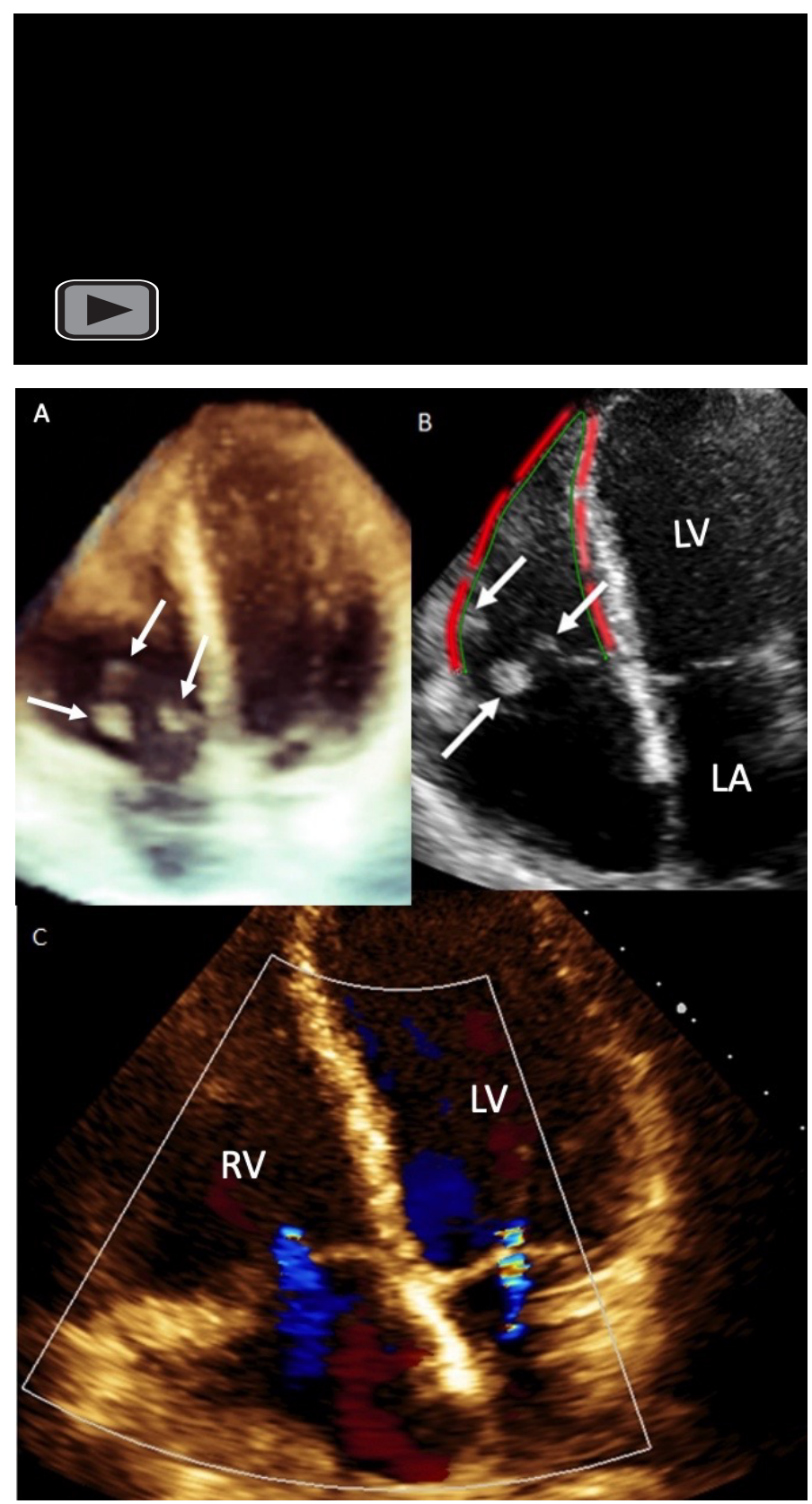

Figure 1. Transthoracic echocardiogram

A- 3D modified four chamber view showing multiple fibroelastomas in the tricuspid valve (white arrows). B- The white arrows are pointing the fibroelastomas in the tricuspid valve in 2D four-chamber view. C-Bidimensional and color Doppler in four chamber view showing mild dilation of the right cavities and mild tricuspid and mitral valve regurgitation. Abbreviations. RV-right ventricle, LV-left ventricle, LA-left atrium.

\section{Discussion}

Tricuspid PFE represents only a small percentage of all the primary heart tumors, which in itself makes this case report a very interesting and infrequent finding. The fact that this patient presented more than one PFE, and that he has remained asymptomatic throughout followup demonstrates an unusual presentation. A case series study that included 725 patients with PFE reported the morphologic and location characteristics of this tumor. Tumors varied from 2 to $70 \mathrm{~mm}$ in size with a mean of $9 \mathrm{~mm}$. Over $80 \%$ of fibroelastomas were found on the heart valves, usually on the left side of the heart (aortic 36\%, mitral 29
$\%$, tricuspid $11 \%$, and pulmonary $7 \%$ ), while the remaining lesions were scattered throughout the atria and ventricles. Multiple tumors were present in 9 percent of patients [11].

The certain etiology remains unknown, although several mechanisms behind the development of PFE have been proposed, but none has been proved scientifically. The most commonly accepted explanation for the origin of papillary fibroelastoma is the microthrombus theory, which posits that these lesions are acquired rather than congenital. The microthrombi, which can themselves dislodge and embolize, are believed to form a nidus for the subsequent progression of excrescences [12].

Most of these tumors do not cause symptoms or produce physical findings. Generally, the fibroelastomas on the left side of the heart cause symptoms that include angina, myocardial infarction, transient ischemic attacks, varying degrees of stroke, and sudden cardiac death. A case series of 162 patients with PFE reported that stroke is the most common symptom in patients with PFE of the mitral valve and myocardial infarction in patients with PFE of the aortic valve [5]. In our case, the differential diagnosis included cardiac tumor and vegetation. Infective endocarditis was excluded based on the absence of fever, no history of intravenous drug abuse and negative blood cultures.

At the present time, transthoracic and transesophageal echocardiography is the most accepted noninvasive diagnostic technique. However, this case remarks the importance of transthoracic echocardiogram for its outstanding definition of consistency and localization of the tumors $[5,13]$.

\section{Conclusion}

Papillary fibroelastomas are rare tumors that account for only $8 \%$ of all benign cardiac tumors [14] and are reported to be typically small and slow growing. This is consistent with the fact that in seven years of close follow-up, our patient has not shown significant changes in the size of the tricuspid PFE on the transthoracic echocardiogram.

Echocardiography was very useful not only as a diagnostic method, but also in making therapeutic decisions.

In our case, a coronary bypass was performed by surgical approach of his multivessel coronary disease and clinical and echocardiographic follow-up of multiple tricuspid fibroelastoma due to its asymptomatic nature.

\section{Highlights}

1. Papillary fibroelastomas are the third most common primary cardiac tumor. Most patients with PFE are clinically asymptomatic.

2. Transesophageal echocardiography is the recommended imaging modality for diagnosis and characterization of PFE, with a good sensitivity and specificity in lesions measuring $2 \mathrm{~cm}$ or more.

3. This case remarks the importance of transthoracic echocardiogram for its outstanding definition of consistency and localization of the tumors.

\section{Funding}

We report no financial support.

\section{Conflicts of interest}

No conflict of interest between the authors. 


\section{Ethical standards}

The authors assert that all procedures contributing to this work comply with the ethical standards of the relevant national guidelines on human experimentation and with the Helsinki Declaration of 1975, as revised in 2008, and has beenapproved by the institutional committees.

\section{References}

1. Burke A (2015) Tumors of the heart and great vessels. Armed Forces Inst. of Pathology, Washington, D.C.

2. Howard RA, Aldea GS, Shapira OM, Kasznica JM, Davidoff R (1999) Papillary fibroelastoma: increasing recognition of a surgical disease. Ann Thorac Surg 68: 18811885. [Crossref]

3. Sydow K, Willems S, Reichenspurner H, Meinertz T (2008) Papillary Fibroelastomas of the Heart. The Thoracic and Cardiovascular Surgeon 56: 9-13.

4. Roberts WC (1997) Primary and Secondary Neoplasms of the Heart. The American Journal of Cardiology 80: 671-682.

5. Sun JP, Asher CR, Yang XS, Cheng GG, Scalia GM, et al. (2001) Clinical and Echocardiographic Characteristics of Papillary Fibroelastomas: A restrospective and porspective study in 162 patients. Circulation 103: 2687-2693. [Crossref]

6. Saad RS, Galvis CO, Bshara W, Liddicoat J, Dabbs DJ (2001) Pulmonary valve papillary fibroelastoma. A case report and review of the literature. Arch Pathol Lab Med 125: 933. [Crossref]
7. Georghiou G, Erez E, Bernardo A Vidne, Dan Aravot (2003) Tricuspid valve papillary fibroelastoma: an unusual cause of intermittent dyspnea. Eur J Cardiothorac Surg 23: 429-431. [Crossref]

8. Davoli G, Bizzarri F, Enrico T, Carone E, Muzzi, L, et al. (2004) Double Papillary fibroelastoma of the aortic valve. Tex Heart Inst $J$ 31: 448. [Crossref]

9. Eslami-Varzaneh F, Brun EA, Sears-Rogan P (2003) An unusual case of multiple papillary fibroelastoma, review of literature. Cardiovasc Pathol 12: 170-173. [Crossref]

10. Meaney JF, Kazerooni EA, Jamadar DA, Korobkin M (1997) CT appearance of the lipomatous hypertrophy of the interatrial septum. AJR Am J Roentgenol 168: 1081. [Crossref]

11. Gowda RM, Khan IA, Nair CK, Mehta NJ, Vasavada BC, et al. (2003) Cardiac papillary fibroelastoma: a comprehensive analysis of 725 cases. Am Heart J 146: 404 410. [Crossref]

12. Malik MF, Sagar K, Wynsen JC, Kenny D (1998) Evolution of a papillary fibroelastoma. J Am Soc Echocardiogr 11: 92-94.

13. Rohani A, Bigdelu L, Nezafati M, Akbari V, Three-dimensional echocardiography of a tricuspid valve papillary fibroelastoma. Journal of the Saudi Heart Association 29: 57-59.

14. Mendes LC, Melo NJ, Souza JB, Correia EB, Zamorano MM, et al. (2012) Fibroelastoma papilífero: relato de sete casos. Arq Bras Cardiol 98: 59-61.

Copyright: $(2021$ Tapia-Sansores M. This is an open-access article distributed under the terms of the Creative Commons Attribution License, which permits unrestricted use, distribution, and reproduction in any medium, provided the original author and source are credited. 